\title{
Fundamental Role of Neurochemicals Aberration in the Pathogenesis of Autism Spectrum Disorders
}

\author{
Nagwa A. Meguid ${ }^{1}$, Hanaa H. Ahmed ${ }^{2 *}$, Manal A. Gad ${ }^{3}$, Olwaya M. Abdel Baki ${ }^{3}$, Samia S. Aziz ${ }^{3}$, Emad F. Eskander ${ }^{2}$ \\ ${ }^{1}$ Department of Research on Children with Special Needs, National Research Centre, Dokki, Giza, Egypt; '2Department of \\ Hormones, National Research Centre, Dokki, Giza, Egypt; ${ }^{3}$ Department of Medical Studies, Faculty of Graduate Studies for \\ Childhood, Ain Shams University, Cairo, Egypt
}

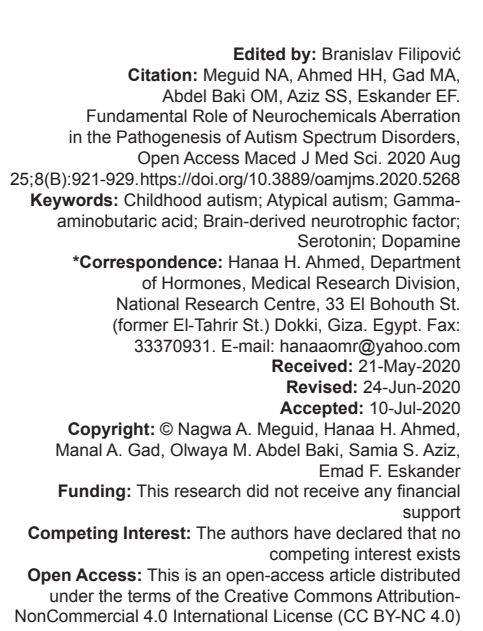

Abstract

AIM: The aim of this research was to establish the perturbation of reliable biomarkers implicated in the pathophysiology of autism to help in the early diagnosis and to be as targets in the treatment of autism spectrum disorders (ASDs) in children and to spotlight into the complex crosstalk between these biomarkers.

PATIENS AND METHODS: This study included 90 autistic children aged from 2 to 7 years old, who were classified into two groups, the atypical autism of 30 children and the childhood autism. The childhood autism group was further divided into mild-moderate autism group and severe autism group each of 30 children. The control group included 30 matched healthy children. All the participants were subjected to full psychiatric examinations, psychological investigations, and biochemical measurements, including gamma-aminobutaric acid (GABA), serotonin, dopamine (DA) in plasma, and brain-derived neurotrophic factor (BDNF) in serum.

RESULTS: The autistic groups showed a highly significant increase in GABA, serotonin, DA, and BDNF levels compared to the control. Of note, the levels of GABA, DA, and BDNF were significantly increased with the increased disease severity. Furthermore, a significant positive correlation between BDNF levels and both GABA and DA levels in the childhood autism group has been recorded.

CONCLUSION: The present clinical setting provides new insight into the fundamental role of BDNF in the brain of autistic children as any alterations of its level due to GABA increment cause change in serotonin and DA levels which have empirical evidence in the pathophysiology of ASD. The results received in this research, create a fertile base for the setup of particular targets in the intervention of this ailment.

\section{Introduction}

The Diagnostic and Statistical Manual of Mental Disorders, $5^{\text {th }}$ Revision (DSM-V) describes autism spectrum disorder (ASD) as a neurological disorder with a spectrum of qualitative impairments in social interaction and in communication as well as restricted stereotyped patterns of behavior, activities, and interests. Autism is growing faster than any other special needs disorders worldwide. The rate of ASD has increased dramatically in the globe by $600 \%$ in the past few decades [1]. The most widely published male-female ratio of autism incidence is $4-5: 1$, lower in people with intellectual disability and higher at the high functioning end. Interestingly, a higher percentage of females have coincident intellectual disabilities [2].

The rational for dismissing peripheral influences on the brain due to the belief that the brain is protected from the events that impinge on the peripheral organs which is being increasingly undermined. The blood brain barrier does not mature until significantly after birth, and its permeability is drastically modulated even in adulthood by factors such as fever and circulating cytokines [3]. As well as, multiple stressors as hypoxia, ammonia, and endotoxin have been confirmed to cause the opening up of tight junctions in the BBB, permitting not only pathogens but also small molecules infiltrate the barrier causes a remarkable increase in membrane permeability in the BBB [4]. Other definite regions of the brain identified as the circumventricular organs fail to develop BBB, so they are potential gates for toxicants if not close of by BBB after 1 year [5]. Furthermore, in humans, about $500 \mathrm{ml}$ of CSF is absorbed into blood daily, making blood appropriate source of neurodegenerative disease biomarkers [6].

Alterations in the neurotransmitters during brain development involve serotonergic and dopaminergic systems which are proposed to be contributed to the pathophysiology of ASD [7]. Researchers have mainly concentrated on mapping biomarkers onto clinically distinct categories, but such categories do not catch the present understanding of the increasingly multidimensional and complex clinical, cognitive, and behavioral phenotype that is connected with autism and its overlap with other disorders [8]. Serotonin, for many years, has been hypothesized that it plays an important role in the pathogenesis of autism. The first support 
that serotonin may have a role in autism derived from studies that measure blood level of serotonin from patients with autism, which found that blood and serum serotonin levels are increased even though these effects differed by age and ethnicity [9]. Dopamine (DA) acts as an essential neurotransmitter in the brain; the dopaminergic effect is mostly exerted over the frontal lobe and basal ganglia. It has been stated that cognitive deficits express changes in these subcortical brain structures with modification of executive functions. These observations propose a role for DA in regulating cognitive functions. A study of Kohls et al. [10] mentioned that individuals with ASD exhibit a reduced response to reward, which derives from striatal dysfunction. ASD is connected with variants in several genes of DA network, as well as those encoding STX1, the DA transporter, DA receptors, and enzymes involved in DA metabolism [11]. Gamma-aminobutaric acid (GABA) represents only $10-15 \%$ of all neuronal populations. Yet, they supply the functional balance, complexity, and computational architecture of neuronal circuits. Adding to its function as an inhibitory neurotransmitter, GABA is as well implicated in neural maturity and circuit development. The GABA neurotransmitter structure has as well been involved in the pathophysiology of ASD, throughout genetic connections with areas coding for GABA-A receptor subunits in families with ASD [12]. $A$ numeral of researches noted different outcomes concerning brain-derived neurotrophic factor (BDNF) values in the serum or brains of autistic individuals. The BDNF levels have been noted to be 3 times as elevated as a control in the basal forebrain of ASD patients [13].

Accordingly, the goals of this work were to justify the aberration of the relevant neurochemical markers contributed in the pathogenesis of ASD and to explore the key actors in this pathologic ailment to facilitate early diagnosis and establishment of targeted therapeutic strategy.

\section{Patients and Methods}

\section{Patients}

The current study was carried out on 90 children diagnosed as childhood autism and atypical autism (case groups) according to the World Health ICD-10 criteria. The study included 66 males and 24 females aged from 2-7 years old. All the diagnosed children frequently attended the outpatient Clinic of Center for Care of Children with Special Needs; Faculty of Post Graduate Studies for Childhood, Ain Shams University, Cairo, Egypt, during the period from January 2014 to January 2015. The atypical autism group consisted of 30 children and the childhood autism group consisted of 60 children. The childhood autism group was further classified into two groups according to the severity of the disorder measured by Childhood Autism Rating Scale (CARS), mild-moderate autism group and severe autism group; each group consisted of 30 children. The study also included 30 healthy children (15 males and 15 females) as a control group, they were age and sex matched and they were healthy schoolchildren from Cairo, Egypt. Depending on Correia et al. [14] who found that Mean \pm SD of the BDNF in $\mu \mathrm{g} / \mathrm{L}$ in autistic and control children are $40.44 \pm 13.87$ and $23.26 \pm 12.34$, respectively, and by considering the power $=0.80$ and $\alpha=0.05$ as well as when using PASS $11^{\text {th }}$, the minimal sample size for an equal size study is released to be 11 in each group [15]. Therefore, we recruited 30 in each group for possible other comparisons.

\section{Inclusion criteria}

The following criteria were included in the study:

- $\quad$ Age from 2 to 7 years.

- $\quad$ Male and female children.

- Medication free for at least 1 month before inclusion in the study.

\section{Exclusion criteria}

The following criteria were excluded from the study:

- $\quad$ Children with autistic feature syndromes as Down syndrome.

- Children with Rett disorder.

- $\quad$ Children with Asperser's disorder.

- $\quad$ Children with childhood disintegrative disorder.

- $\quad$ Children with cerebral palsy.

- $\quad$ Children with central nervous system (CNS) diseases.

- $\quad$ Children with sensory impairments.

- $\quad$ Children with epilepsy in association with autoimmune diseases or any inflammatory conditions.

This study was approved by the "Ethical Committee" of National Research Centre, Giza, Egypt, and the written informed consent was obtained from the parents of the studied patients after explanation of the aim of the study and its possible benefits on their children and other children who have the same conditions (Ethical approval numbers: 11023).

\section{Methods}

All the children included in the study (patients and controls) were subjected to the following: Full psychiatric history and complete psychiatric examination. Each child was submitted to full medical history and clinical examination with particular emphasis on complete neurological examination, and any immune activation such as elevated temperature, 
infections, or inflammatory diseases. Furthermore, EEG with full neurological history was done to exclude the presence of epilepsy in the subjects included in this study. Psychological assessment was performed to confirm the diagnoses of both childhood autism and atypical autism and to detect the severity of the disease using the CARS Second Edition. It is subjectively rated 15 items; relating to people, imitation, emotional response, body use, object use, adaptation to change, visual response, listening response, taste-smell-touch response and use, fear and nervousness, verbal communication, non-verbal communication, activity level, level of consistency of intellectual response, and general impressions. The second edition of CARS expands the test's clinical value, making it more responsive to individuals on the "high functioning" end of autism spectrum disorders. The clinician rates the individual on each item, using a 4-point rating scale. Ratings are based on frequency of the behavior in question, its intensity, peculiarity, and duration [16]. The scores of our patients and control groups are estimated and then categorized in severity according to CARS scores into non-autistic with scores $<25$, autistic features with scores 25-29, mild-moderate autism with scores 30-36, and severe autism with scores 37-60. Vineland Adaptive Behavior Scale (VABS) Second Edition was applied to measure the personal and social skills since birth, diagnosing and classifying mental retardation, and autistic disorder. It assesses adaptive behavior in four domains: Communication, daily living skills, socialization, and motor skills. It also provides a composite score that summarizes the individual's performance across all four domains [17]. The scores of our patients and control children are estimated and their social intelligent quotient (IQ) was detected according to the Vineland IQ scores which are divided into borderline IQ with scores 80-71, mild deficient IQ with scores 70-51, moderate deficient IQ with scores 50-35, severe deficient IQ with scores 34-20, and profound deficient IQ with score $<20$. The low average IQ was estimated with scores $80-90$, the average IQ with scores 90-100, the above average IQ with scores 100-110, and the superior IQ with scores above 110.

\section{Biochemical markers}

From all the fasting children (autistic groups and the control group), $5 \mathrm{ml}$ of blood sample was withdrawn from each child by venous arm puncture and partitioned into two tubes; a plain tube left to clot at room temperature for separation of serum and a heparinized tube for separation of platelet-rich plasma. Both the serum and the platelet-rich plasma were separated by centrifugation at $1800 \times g$ under cooling $\left(4^{\circ} \mathrm{C}\right)$ for $15 \mathrm{~min}$. GABA plasma level was estimated using an enzyme-linked immunosorbent assay (ELISA) kit (Glory Science Co., Ltd., USA) according to the manufacturer's protocol. BDNF serum level was measured using an ELISA kit (Glory Science Co.,
Ltd., USA) according to the manufacturer's procedure. Serotonin (5HT) plasma level was measured using an ELISA kit (Glory Science Co., Ltd., USA) according to the manufacturer's instructions. DA plasma level was measured using an ELISA kit (Glory Science Co., Ltd., USA) according to the manufacturer's manual.

\section{Statistical analysis}

Statistical analysis was performed using the SPSS statistical package software for Windows version 20 (SPSS Inc., Chicago, Illinois, USA). Parametric variables among the controls and the studied patient groups were analyzed using two-tailed unpaired t-test; the t-value measures the size of the difference relative to the variation in your sample data, $\mathrm{T}$ is simply the calculated difference represented in units of standard error.

$$
t(\beta \mathrm{I})=((\beta \mathrm{l})-\beta 0) / \mathrm{s} . e(\beta \mathrm{I})
$$

Where, $\beta 0$ is a non-random, known constant which may or may not match the actual unknown parameter value $\beta$, and s.e $(\beta \mathrm{I})$ is the standard error of the estimator $(\beta \mathrm{I})$ for $\beta$.

Qualitative variables were assessed by the Chi-square test. $p<0.05$ (degree of freedom, df = 95\%) was considered significant difference and $p<0.01$ (degree of freedom, df $=99 \%$ ) was considered highly significant difference. Pearson's correlation coefficient $r$ measures the strength and direction of a linear relationship between two variables on a scatter plot. The value of $r$ is always between +1 and -1 .

\section{Results}

This study included 90 male and female autistic children ( $73.3 \%$ of males and $26.7 \%$ of females). The data in Table 1 show that most of the autistic children in both the atypical autism group (70\%) and the childhood autism group $(75 \%)$ are males versus the control group (50\%) and the females are more likely to have

Table 1: Demographic data of the atypical autism group, childhood autism group, and the control groups

\begin{tabular}{|c|c|c|c|c|c|c|}
\hline \multirow[t]{2}{*}{ Description } & \multicolumn{2}{|c|}{$\begin{array}{l}\text { Control } \\
\text { No. } 30 \\
\end{array}$} & \multicolumn{2}{|c|}{$\begin{array}{l}\text { Atypical autism } \\
\text { No. } 30\end{array}$} & \multicolumn{2}{|c|}{$\begin{array}{l}\text { Childhood autism } \\
\text { No. } 60\end{array}$} \\
\hline & $\mathrm{n}$ & $\%$ & $\mathrm{n}$ & $\%$ & $\mathrm{n}$ & $\%$ \\
\hline Male & 15 & 50 & 21 & 70 & 45 & 75 \\
\hline Female & 15 & 50 & 9 & 30 & 15 & 25 \\
\hline Early onset & - & - & 15 & 50 & 37 & 61.7 \\
\hline Regressed onset & - & - & 15 & 50 & 23 & 38.3 \\
\hline Family history & - & - & 16 & 53.3 & 27 & 45 \\
\hline Autism & - & - & 6 & 20 & 8 & 13.3 \\
\hline Mental disord. & - & - & 7 & 23.3 & 15 & 25 \\
\hline Both & - & - & 3 & 10 & 4 & 6.7 \\
\hline Disruptive behav. & 5 & 16.7 & 29 & 96.7 & 60 & 100 \\
\hline Hyperactivity & 4 & 13.3 & 24 & 80 & 48 & 80 \\
\hline Impulsivity & 6 & 20 & 24 & 80 & 52 & 86.7 \\
\hline Temper tantrum & - & - & 12 & 40 & 37 & 61.7 \\
\hline Self-injurious & - & - & 6 & 20 & 27 & 45 \\
\hline Aggressiveness & - & - & - & - & 7 & 11.7 \\
\hline GIT problems & 6 & 20 & 24 & 80 & 52 & 86.7 \\
\hline Sleep problems & 4 & 13.3 & 11 & 36.7 & 42 & 70 \\
\hline
\end{tabular}


atypical autism (30\%) than childhood autism (25\%). Early onset of autism is more common in childhood autism group $(61.7 \%)$ versus the atypical autism group $(50 \%)$. However, regressed onset is more prevalent in atypical autism group (50\%) than childhood autism group (38.3\%). Family history of mental disorders is more common in atypical autism group (53.3\%) versus the childhood autism group (45\%). The disruptive behaviors are almost equally common in both atypical autism group $(96.7 \%)$ and childhood autism group $(100 \%)$ relative to control group (16.7\%). Likewise, the GIT problems are slightly prevailing in the childhood autism group $(86.7 \%)$ versus both the atypical autism group (80\%) and the control group (20\%). In contrast, the sleep problems are highly common in the childhood autism group $(86.7 \%)$ relative to both atypical autism group (36.7\%) and the control group (13.3\%).

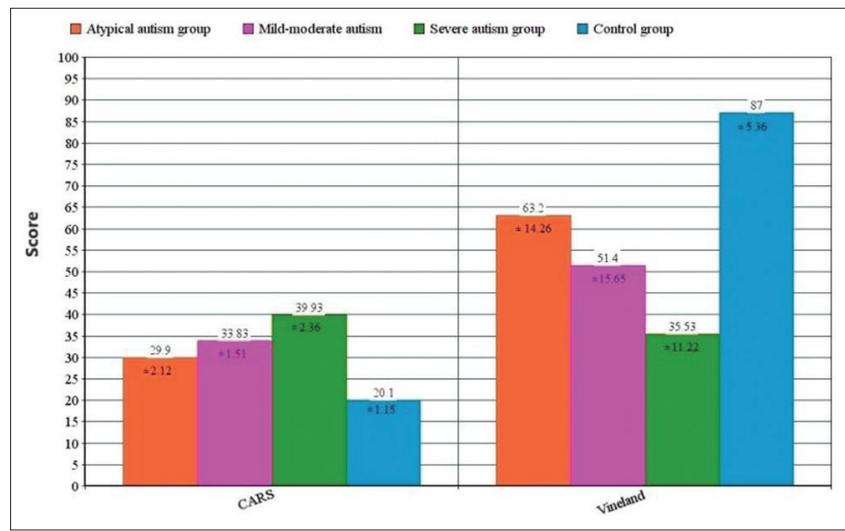

Figure 1: Comparison between atypical autism group and the childhood autism groups (mild-moderate autism and severe autism) and control group according to their scores of psychological tests

The findings in Figure 1 show a highly significant increase regarding to CARS scores in the severe autism group, the mild-moderate autism group, and the atypical autism group ( $p<0.0001)$ compared to the control group. Meanwhile, highly significant decrease regarding to Vineland scores is recorded in the severe autism group, the mild-moderate autism group and the atypical autism group $(P<0.0001)$ versus the control group. Furthermore, the results show a highly

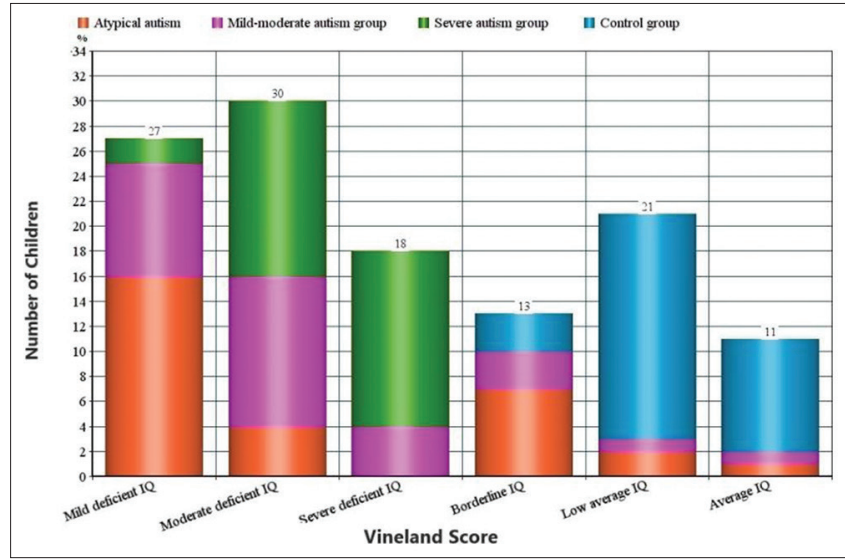

Figure 2: Classification of the autism groups according to Vineland scores significant difference regarding CARS and Vineland scores between the severe autism group and both the atypical autism and the mild-moderate autism groups $(p<0.0001)$.

The results in Figure 2 show that 16 children in the atypical autism group (53.3\%) have Vineland scores of mild deficient IO, 4 children (13.3\%) have scores of moderate deficient IQ, 7 children $(23.3 \%)$ have scores of borderline IQ, 2 children (6.66\%) have a low average IQ, and 1 child (3.33\%) has an average $I Q$, with no scores in severe deficient IQ. While in the mildmoderate autism group, 9 children $(30 \%)$ have scores of mild deficient IQ, 12 children $(40 \%)$ have scores of moderate deficient IQ, 4 children $(13.3 \%)$ have scores of severe deficient IQ, 3 children (10\%) have borderline $I Q$, 1 child $(3.33 \%)$ has scores of low average IQ, and 1 child $(3.33 \%)$ has scores of average IQ. In the severe autism group, 2 children (6.66) have a mild deficient IQ, 14 children (46.7) have a moderate deficient IQ, and 14 children $(46.7 \%)$ have a severe deficient IQ with no borderline or low average and average IQ. In the control group, 3 children (10\%) have scores of borderline IQ, 18 children $(60 \%)$ have scores of low average IQ, and 9 children $(30 \%)$ have scores of average IQ.

The records in Figure $3 a$ show a highly significant increase in GABA plasma levels comparing the atypical autism group to the control group ( $p$ $=0.005$ ). However, there is a significant increase in GABA plasma levels in the mild-moderate autism group relative to the atypical autism group $(p=0.049)$. There is also a greatly significant increase in GABA plasma levels comparing the severe autism group to both the mild-moderate and the atypical ( $p<0.0001)$. Regarding the BDNF serum levels (Figure $3 \mathrm{~b}$ ), there is a significant

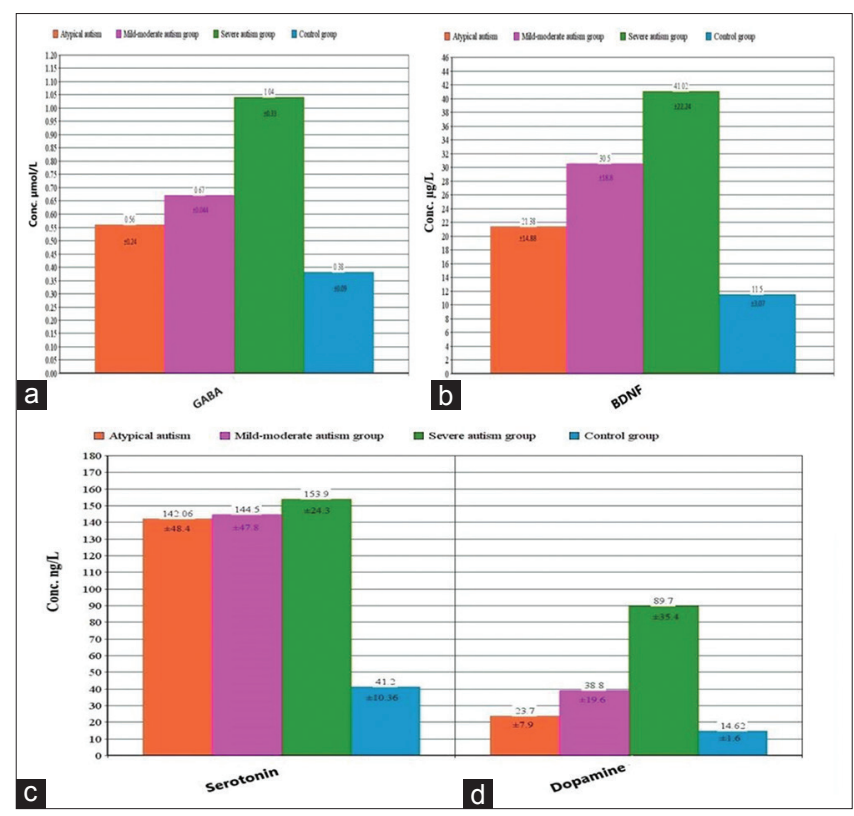

Figure 3: Comparison between atypical autism group, childhood autism groups (mild-moderate autism and severe autism groups), and the control group regarding the biochemical measurements; gamma-aminobutaric acid (a), brain-derived neurotrophic factor (b), serotonin (c), and dopamine (d) 
increase ( $p=0.02$ ) in comparing atypical autism group with the control group. In contrast, there is no significant change in BDNF serum levels between mild-moderate autism group and the atypical autism group ( $p=0.442)$. A highly significant increase in serum BDNF is registered in comparing the severe autism group with the atypical autism group $(p<0.0001)$ and only a significant increase comparing the severe autism group with to the mildmoderate autism group $(p=0.02)$. Data in Figure $3 c$ show that there is a highly significant increase regarding serotonin plasma levels in comparing the atypical autism group with the control group $(p<0.0001)$. The results in Figure $3 d$ show a highly significant increase in DA plasma levels in the atypical autism group versus the control $(p<0.0001)$. Meanwhile, there is no significant difference in comparing all the autism groups with each other as regard serotonin plasma levels (Figure 3c). As regard DA plasma levels, there is a highly significant increase in the mild-moderate autism group relative to the atypical autism group $(p=0.005)$ and also in the severe autism when compared with both the atypical and the mild-moderate autism groups ( $p<0.0001)$ (Figure 3d).

The records in Figure 4 constitute the Pearson's correlation between the CARS test and Vineland test in childhood autism group. The data show a highly significant negative correlation between CARS and Vineland scores $r=-0.568$ (Figure 4a) and a highly significant positive correlation between CARS scores and both GABA $r=0.387$ (Figure $4 b$ ) and DA $r=0.657$ (Figure 4c). The results also show a highly significant negative correlation between Vineland scores and DA $r=-0.452$ (Figure 4d).

The results in Figure 5 indicate the Pearson's correlation between the measured biochemical parameters with each other in childhood autism group. The data show a significant positive correlation
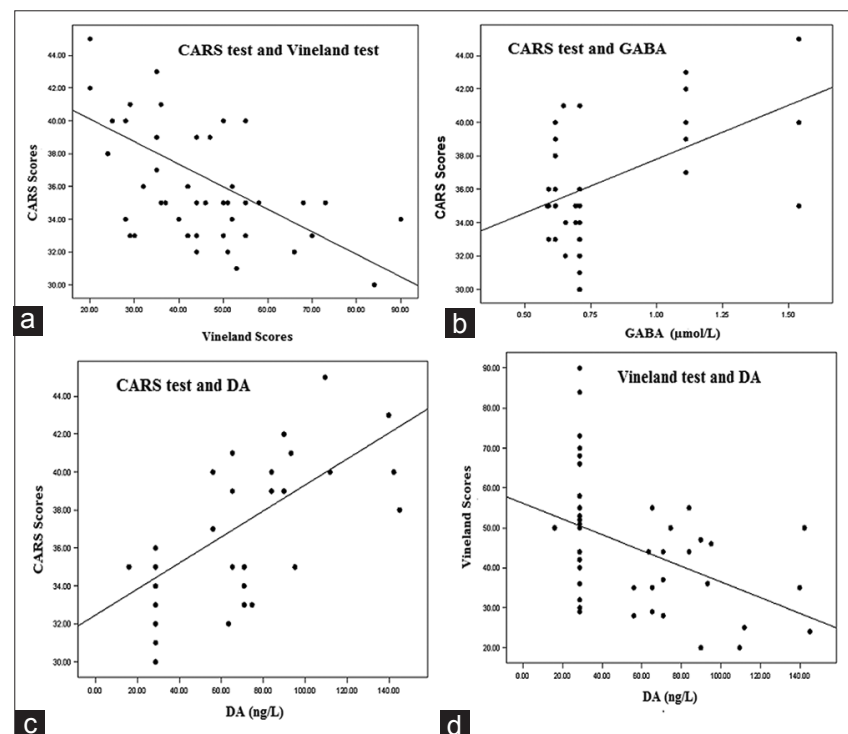

Figure 4: Pearson's correlation between the Childhood Autism Rating Scale (CARS) test and Vineland test (a), CARS test and gammaaminobutaric acid (b), CARS test and dopamine (DA) (c), and Vineland test and DA (d) in childhood autism group between DA and both GABA $r=0.303$ (Figure 5a) and BDNF $r=0.327$ (Figure 5b). However, there is a highly significant positive correlation between GABA and BDNF levels $r=0.387$ (Figure $5 c$ ).

\section{Discussion}

In the current study, $33.3 \%$ of the autistic children are diagnosed as atypical autism and $66.7 \%$ are diagnosed with childhood autism. This is in conformity with Hussein et al. [18] who reported that typical autism is more common than atypical autism in both Saudi and Egyptian groups. Furthermore, the preponderance of autistic patients in both atypical autism and childhood autism groups is boys $70 \%$ and $75 \%$, respectively. This is in keeping with Xiong et al. [19] who reported a higher predominance of boys over girls in association with autistic children

This study demonstrated that $50 \%$ of atypical autism and $38.3 \%$ of childhood autism had regressed onset. This is concurrent with Hansen [20] study which recorded regression in $41 \%$ of subjects with autism; $26 \%$ lost either language or social skills; while 15\% lost both. The present data showed that $53.3 \%$ of atypical autism and $45 \%$ of childhood autism have a family history of autism, other mental disorders, or both. This comes in line with that of Sandin et al. [21] who stated that heritability of ASD and autistic disorder is evaluated to be approximately $50 \%$. Most of the autistic children in this study had disruptive behaviors, $96.7 \%$ in atypical autism and $100 \%$ in childhood autism versus $16.7 \%$ in the control group. The most frequent disruptive behaviors in the atypical autism and the childhood autism groups, respectively, were hyperactive (80\%)

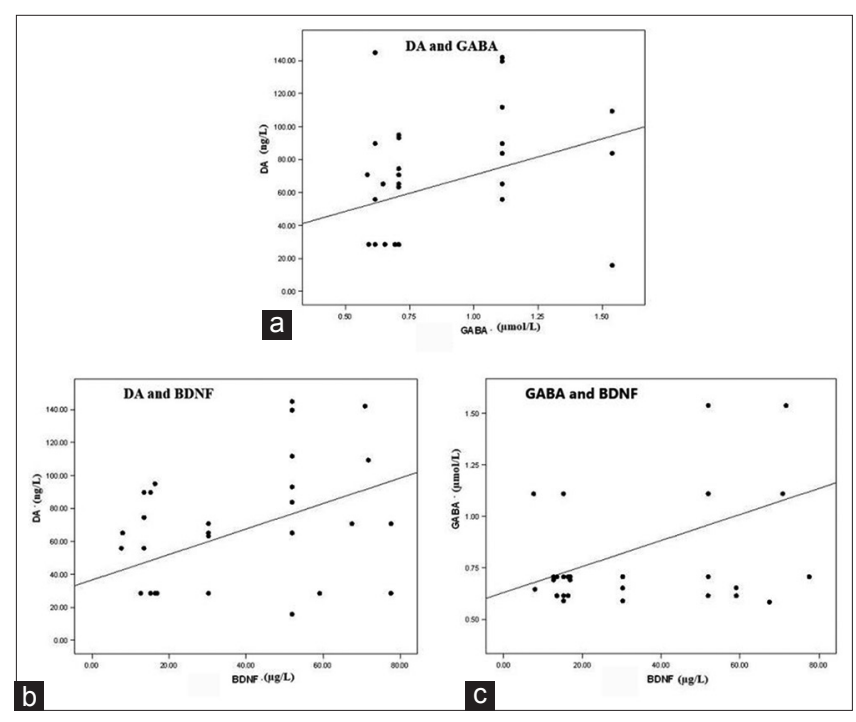

Figure 5: Pearson's correlation between the measured biochemical markers, dopamine (DA) and gamma-aminobutaric acid (GABA) (a), $D A$ and brain-derived neurotrophic factor (BDNF) (b), and GABA and $B D N F(c)$ in childhood autism group 
in both groups, impulsivity (80\%), (86.7\%), temper tantrum $(40 \%),(61.7 \%)$, self-injurious $(20 \%),(45 \%)$, and aggressiveness $(0 \%),(11.7 \%)$, respectively. These results are in harmony with those of Kats et al. [22] who mentioned that disruptive behaviors frequently manifest themselves in children with ASD and up to $60 \%$ of subjects with autism and an intellectual disability present with difficult-to-manage behaviors, including self-injurious, disruptive, and destructive behaviors. Furthermore, most of the autistic children in the present study had gastrointestinal (GI) problems; $80 \%$ in atypical autism and $86.7 \%$ in childhood autism versus $20 \%$ in the control group. These findings are paralleled to the recent report by Lefter et al. [23] who stated that the increased prevalence of $\mathrm{Gl}$ symptoms in ASD patients indicating that $\mathrm{Gl}$ dysfunctions are of particular relevance in ASD. These various $\mathrm{GI}$ manifestations are related to the autonomous nervous system affecting the parasympathetic tone and stress responsiveness as well as abnormal dynamics of neurohormones such as serotonin or GABA. Research based on parental note recommends that autistic children are more liable to have sleep problems than children with other developmental disorders and children with no developmental diagnosis [24]. This finding is in accordance with the results of the present study which demonstrated that $36.7 \%$ of atypical autism and $70 \%$ of childhood autism versus $13.3 \%$ of the control group had sleep problems.

In the present investigation, measuring the autism severity was done using CARS which demonstrated that in the atypical autism group, $100 \%$ had autistic features, while in the childhood autism group, $50 \%$ had mild-moderate autism and the rest $50 \%$ had severe autism. These results match the previous researches indicating that CARS total score changes significantly between the two groups, with autistic disorder being significantly elevated than atypical autism [25]. Results of the present study echo those of the previous findings indicating that the CARS total score changes significantly by diagnostic group, as a significantly higher total CARS scores have been found in the severe autism group versus the mildmoderate autism group when compared both with the atypical autism group and the control group. The clinical significant differences reported in CARS total scores among the diagnostic groups are congruent with the results of Chlebowski et al. [26] who supported the utilization of CARS as a stable measure of autism severity.

The current results also showed that there are significantly lower scores in Vineland Adaptive Behavior Scale with the elevation of the disease severity. Greatly significant $(p<0.0001)$ low scores have been detected by comparing the severe autism group with the mildmoderate autism group and in comparing both to the atypical autism group and the control group. These results are in concert with those of Paul et al. [27] who commented that individuals with atypical autism scored higher than those with autism in Vineland Adaptive Behavior Scale. This showed parallelism with our results, as it has been detected a significant $(p<0.0001)$ negative correlation between CARS and Vineland IQ in childhood autism group.

The present study showed an increase of GABA plasma level with the increase in the severity of the disease. It has been observed a marked significant increase $(p<0.0001)$ in the plasma level of GABA in comparing both the mild-moderate autism and the severe autism groups to both the atypical autism group and the control group and in comparing the severe to the mild-moderate autism groups. Furthermore, it has been found a marked significant ( $p=0.002$ ) positive correlation between GABA levels and CARS scores in the childhood autism group. These results are comparable to those in the previous study done by Enticott et al. [28] which demonstrated the significantly elevated level of plasma GABA in patients with autism in comparison to the controls. These investigators stated that GABA, as the major inhibitory transmitter in the CNS, has been implicated in the pathophysiology of ASDs. As well, Mendez et al. [29] proved the correlation between elevated plasma GABA and severity of autism using CARS and that severe autistics show higher plasma GABA levels relative to mild-moderate patients. There are many evidences supporting the function of GABA in the etiology or pathophysiology of autistic disorders. One influential theory proposed by Casanova et al. [30] is that inhibitory GABA signaling inside and among cortical minicolumns are altered due to the decreasing in the neuropil which divides closest minicolumns leading to information processing that tends to demonstrate stronger than normal inequity between connected stimuli rather than generalization between them. This may suggest why patients with autism demonstrate a preference for precise similarity (e.g., the same daily routines, the same behaviors, and interests), as just precisely the identical stimuli would be detected as similar. It may also clarify sensory hypersensitivities and the occasional existence of superior "savant" abilities in a narrow domain.

The tabulated results pointed to the heightened of BDNF level with the increase in the severity of the disease, as it has been found a marked significant increase $(p=0.002)$ in BDNF serum level in severe autism group comparative to the atypical autism group and a significant increase $(p=0.02)$ in its level in comparison to the mild-moderate autism group. In addition, an insignificant increase $(p=0.4)$ in BDNF serum level is recorded in comparing the mild-moderate autism group to the atypical autism group. These results are consistent with the study of Zhang et al. [31] which measured serum level of BDNF in Chinese patients with ASD, and the severity of ASD was evaluated with CARS score. Their results suggested that the median serum BDNF levels are significantly $(p<0.0001)$ higher in autistic children as compared to normal cases, and 
they discovered that its higher levels may be considered as an independent risk factor of ASD. Wang et al. [32] also detected that the mean serum BDNF levels are significantly $(p<0.0001)$ upregulated in children with ASD in comparison to the control cases and serum BDNF levels may be correlated independently with the severity of ASD. BDNF can influence synaptic inhibition by reducing the surface constancy and expression of GABA type A [33]. Powers et al. [34] observed that BDNF exposure reduces the inhibitory effects of GABA on parvocellular neuroendocrine neurones from the paraventricular nucleus of the hypothalamus and that oral administration of the amino acid/inhibitory neurotransmitter GABA reportedly increases the resting serum growth hormone $(\mathrm{GH})$ concentrations by $400 \%$. One of the mechanisms mediating BDNF is the $\mathrm{GH}$. The role of BDNF in the pathophysiology of ASD was defined by Almeida et al. [35] who stated that activating BDNF signaling can sometimes be pathogenic. For example, adult transgenic mice overexpressing BDNF are prone to seizures and increasing of BDNF levels enhances pain sensitivity. Moreover, during early embryonic brain development, the cell cycle parameters of proliferating neuroblasts and the laminar fate of their progeny are extremely sensitive to either increased or decreased BDNF signaling. These explanations greatly support the present study, which showed a highly significant ( $p=0.002$ ) positive correlation between BDNF and GABA level in the childhood autism group, which both their levels are increased with the severity of the disease.

In this study, plasma level of serotonin was found to be highly significantly elevated in comparing all the autistic groups versus the control group, but insignificant change of its level has been detected by comparing all the autistic groups with each other. These results fit similar findings recorded by Chandana et al. [36] who found a high proportion of children with autism exhibiting elevated plasma serotonin levels and specific alterations in serotonin biosynthesis. The observed increase of serotonin plasma level in all autistic groups under study could be referred to the increased of BDNF observed in these patients, as BDNF has also been found to regulate serotonergic neurotransmission in vitro. In addition, BDNF administration has been revealed to increase the synthesis of serotonin in vivo [37]. Thereby, serotonin has a potential role in the pathogenesis of autism. In addition, blood serotonin levels have been discovered to correlate inversely with verbal $I Q$ and to correlate positively with the severity of autism [38].

In the current approach, plasma concentration of DA showed insignificant $(p=0.2)$ increase in the atypical autism group relative to the healthy control group, while it showed significant increase with the severity of the disease, as it showed highly significant increase in both the mild-moderate $(p=005)$ and the severe autism groups $(p<0.0001)$ in respect to the atypical autism group and also in comparing the severe autism group to the mild-moderate autism group. These results are in accordance with those of El-Ansary et al. [39] who reported that Saudi autistic patients have a significantly higher level of DA compared to healthy subjects. One explanation for the significant elevation of DA and increased its level in correlation with the severity of the disease may be due to BDNF, which induces long-term changes in brain function by influencing the reactions of its target neurons to DA [40]. Hence, dysregulation of the function of BDNF coupled with dysfunction of DA, a neurotransmitter that is critical for so many cognitive and motor skills may underlie the expression of specific symptoms connected with autism. It is well documented that the dopaminergic system has been correlated with speech and communication abilities which are defective in autism. DA also modulates and reduces behavioral reactions to changes in the social environment [41]. This is in consistent with the current results as it has been found a significant positive correlation $(p=0.01)$ between DA and both BDNF and GABA, and a highly significant ( $p=0.002$ ) positive correlation between DA levels and CARS scores in the childhood autism group, which all are increased with the severity of the disease.

\section{Study limitations}

This study has some potential limitations; the sample size was relatively small because it was so difficult to find children not taking any medications for at least 1 month as in our exclusion criteria since any neurological or psychiatric drugs will affect the neurochemical level that we were measuring. As well, it was so difficult to withdraw blood from autistic children at this young age from 2 to 7 years as almost all of them were having severe behavioral problems as hyperactivity and temper tantrums. Moreover, for the clinical work, we had some limitations as more phenotypes of autism as Asperger disorder should be added in the study population to be compared with childhood autism and atypical autism groups but Asperger disorder is not a common disorder in Egypt.

\section{Conclusion}

The data of this study speak for the importance of BDNF in the brain where any prolonged perturbations in its level due to the increase of GABA most probably lead to aberration in serotonin and DA, which are well key actors in the pathophysiology of many psychiatric diseases in general and ASD in particular. In light of these evidences, it should reconsider the crosstalk between the brain neurochemicals committed in ASD to set up a line of targeted therapy for this neurodevelopmental disorder. 


\section{Recommendations}

- Mapping the neurochemical markers onto clinically distinct categories, to better understanding the multidimensional and complex clinical, cognitive, and behavioral phenotype that are connected with autism and its overlap with other disorders. in the pathophysiology of autism to spotlight into the complex crosstalk between these biomarkers.

\section{Acknowledgments}

We acknowledge with gratitude the patients, the outpatient Clinic of Center for Care of Children with Special Needs; Faculty of Graduate Studies for Childhood, Ain Shams University, and the Directors of National Research Centre for their encouragement and support.

\section{References}

1. Stephen W, Dawson G. Risk factors for autism: Translating genomic discoveries into diagnostics. Hum Genet. 2011;130(1):123-48. https://doi.org/10.1007/s00439-011-1037-2 PMid:21701786

2. Lai M, Lombardo M, Auyeung B, Chakrabarti B, Baron-Cohen S Sex/gender differences and autism: Setting the scene for future research. J Am Acad Child Adolesc Psychiatry. 2015;54(1):1124. https://doi.org/10.1016/j.jaac.2014.10.003 PMid:25524786

3. Herbert M, Anderson M. An expanding spectrum of autism models, from fixed developmental defects to reversible functional impairments. In: Zimmerman A, editor. Autism, Current Theories and Evidence. Totowa, New Jersey: Human Press; 2008. p.42963. https://doi.org/10.1007/978-1-60327-489-0_20

4. Seneff S, Lauritzen A, Davidson R, Lentz-Marino L. Is encephalopathy a mechanism to renew sulfate in autism. Entropy. 2013;15:372-406. https://doi.org/10.3390/e15010372

5. McGinnis W, Miller V, Audhya T, Edelson S. Neurotoxic brainstem impairment as proposed threshold event in autistic regression. In: Chauhan A, Chauhan V, Brown T, editors. Autism, Oxidative Stress, Inflammation, and Immune Abnormalities. London, New York: Taylor and Francis Group, CRC Press; 2010. p. 154-68. https://doi.org/10.1201/9781420068870-c9

6. Chauhan A, Chauhan V, Brown W. Cohen I. Oxidative stress in autism: Increased lipid peroxidation and reduced serum levels of ceruloplasmin and transferrin the antioxidant proteins. Life Sci. 2004;75(21):2539-49. https://doi.org/10.1016/j.lfs.2004.04.038 PMid:15363659

7. Chugani D. Understanding alterations during human brain development with molecular imaging: role in determining serotonin and GABA mechanisms in autism. In: Blatt J, editor. The Neurochemical Basis of Autism from Molecules to Minicolumns. New York, London: Spring Science, Business Media; 2010. p.

83-93. https://doi.org/10.1007/978-1-4419-1272-5_6

8. Elsabbagh M, Johnson M. Getting answers from babies about autism. Trends Cogn Sci. 2010;14(2):81-7. https://doi. org/10.1016/j.tics.2009.12.005

PMid:20074996

9. Lam K, Aman M, Arnold L. Neurochemical correlates of autistic disorder: A review of literature. Res Dev Disabil. 2006;27(3):254-89.

PMid: 16002261

10. Kohls G,Thonessen H, Bartly G, Grossheinrich N, Fink G, Herpertz-Dahlmann B, et al. Differentiating neural reward responsiveness in autism versus ADHD. Dev Cogn Neurosci. 2014;10:104-16. https://doi.org/10.1016/j.den.2014.08.003 PMid:25190643

11. Cartier E, Hamilton P, Belovich AN, Shekar A, Campbell N Saunders $\mathrm{C}$, et al. Rare autism associated variants implicate syntaxin 1(STX1 R26Q) phosphorylation and dopamine transporter (hDAT R51W) in dopamine neurotransmission and behaviours. EBioMedicine. 2015;2(2):135-46. https://doi. org/10.1016/j.ebiom.2015.01.007

PMid:25774383

12. Collins A, Ma D, Whitehead P, Martin E, Wright H, Abramson R, et al. Investigation of autism and GABA receptor subunit genes in multiple ethnic groups. Neurogenetics. 2006;7(3):167-74. https://doi.org/10.1007/s10048-006-0045-1

PMid:16770606

13. Shimizu E, Hashimato K, Okamuran N, Koike K, Komatsu N, Kumakiri $\mathrm{C}$, et al. Alterations of serum levels of brain derived neurotrophic factor (BDNF) in depressed patients with or without antidepressants. Biol Psychiatry. 2003;54(1):70-5. https://doi. org/10.1016/s0006-3223(03)00181-1

PMid: 12842310

14. Correia C, Coutinho A, Sequeira A, Sousa I, Lourenc L, Venda $\mathrm{O}$, et al. Increased BDNF levels and NTRK2 gene association suggest a disruption of BDNF/TrkB signaling in autism. Genes Brain Behav. 2010;9(7):841-8. https://doi. org/10.1111/j.1601-183x.2010.00627.x PMid:20662941

15. Hintze J. PASS 11, Power Analysis and Sample Size User Guide 3. Kaysville, Utah: NCSS; 2011.

16. Schopler E, Van Bourgondien M, Wellmann G. The Childhood Autism Rating Scale. $2^{\text {nd }}$ ed. Los Angeles, California: Western Psychological Services; 2010. https://doi. org/10.1177/0734282911400873

17. Sparrow S, Balla D, Cicchetti D. Vineland Adaptive Behaviour Scales. Circle Pines: Minn American Guidance Service; 1984.

18. Hussein H, Taha G, Almanasef A. Characteristics of autism spectrum disorders in a sample of Egyptian and Saudi patients: Transcultural cross sectional study. Child Adolesc Psychiatry Ment Health. 2011;5:34. https://doi.org/10.1186/1753-2000-5-34 PMid:22051160

19. Xionga X, Liua D, Hea W, Shenga X, Zhouc W, Xiea D, et al Identification of gender-related metabolic disturbances in autism spectrum disorders using urinary metabolomic. Int J Biochem Cell Biol. 2019;115:105594.

PMid:31449876

20. HansenR, Ozonoff S, Krakowiak P, Angkustsiri K, Jones C Deprey L, et al. Regression in autism: Prevalence and associated factors in the charge study. Ambul Pediatr. 2008;8(1):25-31. https://doi.org/10.1016/j.ambp.2007.08.006 PMid: 18191778

21. Sandin S, Lichtenstein $P$, Kuja-Halkola $R$, Larsson $H$ Hultman C, Reichenberg A. The familial risk of autism. JAMA. 2014;311(17):1770-7. https://doi.org/10.1001/jama.2014.4144 PMid:24794370 
22. Kats D, Payne L, Parlier M, Piven J. Prevalence of selected clinical problems in older adults with autism and intellectual disability. J Neurodev Disord. 2013;5(1):27. https://doi. org/10.1186/1866-1955-5-27 PMid:24066979

23. Lefter R, Ciobica A, Timofte D, Stanciu C, Trifan A. Prevalence of selected clinical problems in older adults with autism and intellectual disability. Medicina. 2020;56:11. https://doi. org/10.3390/medicina56010011

24. Polimeni M, Richdale A, Francis A. A survey of sleep problems in autism, asperger's disorder and typically developing children. J Intellect Disabil Res. 2005;49(4):260-8. https://doi. org/10.1111/j.1365-2788.2005.00642.x

PMid:15816813

25. Tachimori $\mathrm{H}$, Osada $\mathrm{H}$, Kurita $\mathrm{H}$. Childhood autism rating scale-Tokyo version for screening pervasive developmental disorders. Psychiatry Clin Neurosci. 2003;57(1):113-8. https:// doi.org/10.1046/j.1440-1819.2003.01087.x

PMid:12519463

26. Chlebowski C, Green J, Barton M, Fein D. Using the childhood autism rating scale to diagnose autism spectrum disorders. J Autism Dev Disord. 2010;40(7):787-99. https://doi.org/10.1007/ s10803-009-0926-x

PMid:20054630

27. Paul R, Miles S, Cicchetti D, Sparrow S, Klin A, Volkmar $F$, et al. Adaptive behavior in autism and pervasive developmental disorder-not otherwise specified: Microanalysis of scores on the vineland adaptive behavior scales. J Autism Dev Disord. 2004;34(2):223-8. https://doi. org/10.1023/b:jadd.0000022612.18116.46

PMid: 15162940

28. Enticott P, Kennedy H, Rinehart N, Tonge B, Bradshaw J, Fitzgerald P. GABAergic activity in autism spectrum disorders: An investigation of cortical inhibition via transcranial magnetic stimulation. Neuropharmacology. 2013;68:202-9. https://doi. org/10.1016/j.neuropharm.2012.06.017 PMid:22727823

29. Mendez M, Horder J, Myers J, Coghlan S, Stokes P, Erritzoe $D$, et al. The brain GABA-benzodiazepine receptor alpha-5 subtype in autism spectrum disorder: A pilot positron emission tomography study. Neuropharmacology. 2013;68:195-201. https://doi.org/10.1016/j.neuropharm.2012.04.008 PMid:22546616

30. Casanova M, Buxhoeveden D, Gomez J. Disruption in the inhibitory architecture of the cell minicolumn: Implications for autism. Neuroscientist. 2003;9(6):496-507. https://doi. org/10.1177/1073858403253552 PMid:14678582

31. Zhang $Q$, Jiang $L$, Kong $L, L u Y$. Serum brain-derived neurotrophic factor levels in Chinese children with autism spectrum disorders: A pilot study. Int J Dev Neurosci. 2014;37:65-8. https://doi. org/10.1016/j.ijdevneu.2014.06.013 PMid:24984148

32. Wang M, Chen H, Yu T, Cui G, Jiao A, Liang H. Increased serum levels of brain-derived neurotrophic factor in autism spectrum disorder. Neuroreport. 2015;26(11):638-41. https:// doi.org/10.1097/wnr.0000000000000404

\section{PMid:26103118}

33. Jovanovic J, Thomas P, Kittler J, Smart T, Moss S. Brainderived neurotrophic factor modulates fast synaptic inhibition by regulating GABA (A) receptor phosphorylation, activity, and cell-surface stability. J Neurosci. 2004;24(2):522-30. https://doi. org/10.1523/jneurosci.3606-03.2004

PMid:14724252

34. Powers M, Yarrow J, McCoy S, Borst S. Growth hormone isoform responses to GABA ingestion at rest and after exercise. Med Sci Sports Exerc. 2008;40(1):104-10. https://doi.org/10.1249/ mss.0b013e318158b518

PMid:18091016

35. Almeida L, Roby C, Krueger B. Increased BDNF expression in fetal brain in the valproic acid model of autism. Mol Cell Neurosci. 2014;59:57-62. https://doi.org/10.1016/j.mcn.2014.01.007 PMid:24480134

36. Chandana S, Behen M, Juhasz C, Muzik O. Significance of abnormalities in developmental trajectory and asymmetry of cortical serotonin synthesis in autism. Int $\mathrm{J}$ Dev Neurosci. 2005;23(2-3):171-82. https://doi.org/10.1016/j. ijdevneu.2004.08.002

PMid:15749243

37. Goggi J, Pullar I, Carney S, Bradford H. Modulation of neurotransmitter release induced by brain-derived neurotrophic factor in rat brain striatal slices in vitro. Brain Res. 2002;941(12):34-42. https://doi.org/10.1016/s0006-8993(02)02505-2 PMid: 12031545

38. Cook E, Leventhal B, Heller W, Metz J, Wainwright M, Freedman D. Autistic children and their first degree relatives: Relationships between serotonin and norepinephrine levels and intelligence. J Neuropsychiatry Clin Neurosci. 1990;2(3):26874. https://doi.org/10.1176/jnp.2.3.268

\section{PMid:2136085}

39. El-Ansary A, Bacha A, Ayahdi L. Relationship between chronic lead toxicity and plasma neurotransmitters in autistic patients from Saudi Arabia. Clin Biochem. 2011;44(13):1116-20. https:// doi.org/10.1016/j.clinbiochem.2011.06.982

\section{PMid:21763301}

40. Guillin O, Diaze J, Carroll P, Griffon N, Schwartz J, Sokoloff P. BDNF controls dopamine D3 receptor expression and triggers behavioural sensitization. Nature. 2001;411(6833):86-9. https:// doi.org/10.1038/35075076

\section{PMid:11333982}

41. Alabdali A, Al-Ayadhi L, El Ansary A. A key role for an impaired detoxification mechanism in the etiology and severity of autism spectrum disorders. Behav Brain Funct. 2014;10:14. https://doi. org/10.1186/1744-9081-10-14

PMid:24776096 